\title{
Prevalencia de la enfermedad de Pompe entre pacientes con elevación de creatinin-kinasa (CK)
}

\author{
J. Marin, V. Scaglioni, J. Zacariaz, F. Vergara, J. Rosa, E. R. Soriano \\ Sección Reumatología, Servicio Clínica Médica, Hospital Italiano de Buenos Aires. Instituto Universitario, Hospital Italiano de Buenos Aires. \\ Fundación PM Catoggio para el Progreso de la Reumatología.
}

\begin{abstract}
Resumen
Introducción: La prevalencia exacta de la enfermedad de Pompe en la población general es desconocida, y la frecuencia estimada varía entre las diferentes formas clínicas y grupos étnicos. Existe un gran número de pacientes con elevación de enzimas musculares que no tienen un diagnóstico definitivo. Un porcentaje de ese grupo de pacientes podría tener enfermedad de Pompe.

Objetivos: 1) Determinar la prevalencia de la enfermedad de Pompe entre pacientes mayores de un año de edad, con elevación persistente de creatin-kinasa (CK) de causa desconocida. 2) Describir las manifestaciones clínicas y demográficas de los pacientes con CPK elevada.

Materiales y métodos: Se incluyeron a todos los pacientes con aumento de CK en sangre, definida como la detección de al menos un valor $>500 \mathrm{UI} / \mathrm{L}$ y otro mayor de $200 \mathrm{UI} / \mathrm{L}$, durante un período mínimo de 1 año, entre los años 2010-2013, tomados de la base de datos del laboratorio del Hospital Italiano de Buenos Aires. Se excluyeron a los pacientes bajo tratamiento actual con agentes hipolipemiantes; pacientes tratados con agentes hipolipemiantes, en quienes la CK no se normalizó luego de suspender el tratamiento; pacientes tratados con agentes hipolipemiantes que interrumpieron el tratamiento durante un tiempo menor de 9 meses antes del período de inclusión y pacientes con enfermedad muscular inflamatoria (pacientes con criterios diagnósticos probables o definidos de Bohan \& Peter). Los pacientes con diagnóstico probable de miositis por cuerpo de inclusión (biopsia no típica) podían ser incluidos. Se les realizó evaluación clínica (medición de la fuerza muscular), cuestionario sobre síntomas musculares y se les extrajo sangre para test enzimático en papel de filtro para enfermedad de Pompe.
\end{abstract}

Resultados: Se revisaron 849 historias clínicas; 199 pacientes cumplieron con los criterios de inclusión de los que 110 pacientes fueron evaluados. Se pudo realizar el test enzimático en papel de filtro para diagnóstico de enfermedad de Pompe en 93 de los 110 pacientes evaluados. A 21 pacientes se les realizó electromiograma y a 10 pacientes biopsia muscular. Se pudo establecer el diagnóstico del aumento de la CK sólo en el 34,4\% de los pacientes. El diagnóstico de enfermedad de Pompe se realizó en 1 paciente, mostrando una prevalencia del 1,07\%. Conclusiones: La prevalencia de enfermedad de Pompe fue similar a la publicada en la literatura. Se pudo hacer el diagnóstico del aumento de CK elevada en un bajo porcentaje de pacientes $(34,4 \%)$.

Palabras clave: enfermedad de Pompe, CPK elevada.

\section{Abstract}

Introduction: The exact prevalence of Pompe disease in the general population is unknown, and the estimated frequency varies among different ethnic groups and clinical forms. A large number of patients with elevated muscle enzymes do not have a definitive diagnosis. A significant percentage of these patients may have a Pompe disease.

Objectives: To determine the prevalence of Pompe disease among patients with persistently elevated CK (over one year) of unknown cause. To describe the demographic and clinical manifestations of the patients with elevated CK.

Patients and methods: We included all patients with increased blood CK, defined as a value $>500 \mathrm{IU} / \mathrm{L}$ and another greater than $200 \mathrm{IU} / \mathrm{L}$ for at least 1 year between 2010 and 2013. Patients were selected from the database of the laboratory of the Italian Hospital of Buenos Aires. We excluded patients under current treatment with lipid-lowering agents; patients who have been treated with lipid-lowering agents, in whom CPK has not normalized after discontinuation of therapy; patients who have been treated with lipid-lowering agents and discontinued for a time less than 9 months before the inclusion period and patients with inflammatory muscle disease: patients with probable or definite criteria diagnoses (Bohan \& Peter criteria). Patients with a diagnosis of probable inclusion body myositis (not typical biopsy) were included. Patients underwent clinical evaluation (measurement of muscle strength), muscle symptoms questionnaire and had blood taken for enzyme test on filter paper for Pompe disease.

Results: 849 medical records were reviewed, 199 patients fulfilled inclusion criteria. We fully evaluated 110 patients, of whom 93 had the enzyme test on filter paper for Pompe performed. Electromyogram was performed in twenty-one patients and muscle biopsy was done in 10 . We could make the diagnosis only in $34.4 \%$ of the patients. One patient was diagnosed with Pompe disease, giving a prevalence of $1.07 \%$.

Conclusions: We found a prevalence of Pompe disease similar to that reported in other studies. Even after a careful review of clinical data, the cause of elevated CK was clear in only a minority of patients.

Key words: Pompe disease, elevated CK. 


\section{Introducción}

La enfermedad de Pompe es una enfermedad caracterizada por el depósito de glucógeno tipo II, secundaria al déficit de maltasa ácida. Es un desorden autosómico recesivo del metabolismo del glucógeno, causado por la deficiencia de la enzima ácida lisosomal alfa-glucosidasa $(\mathrm{AAG})^{1,2}$. La presentación clínica abarca un rango de fenotipos; comúnmente se la clasifica en una forma infantil y otra de comienzo tardío ${ }^{3,4}$. Por definición, la forma de comienzo tardío es aquella que aparece después del año de vida $^{3,4}$, con afección lentamente progresiva de la musculatura esquelética, llegando a la debilidad y finalmente a la insuficiencia respiratoria. La causa de muerte de esta última forma de presentación clínica se debe habitualmente a las complicaciones respiratorias ${ }^{1-5}$.

Hasta el año 2006 el tratamiento de la enfermedad de Pompe era solamente paliativo ${ }^{4,6}$. El descubrimiento de la terapia de reemplazo con la enzima alfa glucosidasa (Myozyme; Genzyme corporation, Cambridge, MA, USA) representó el primer tratamiento efectivo y específico de la enfermedad ${ }^{7-9}$. El diagnóstico requiere de un amplio conocimiento de sus manifestaciones clínicas y de un test rápido y confiable que pueda medir la actividad enzimática de la AAG, para confirmar su deficiencia. Se han desarrollado ensayos que miden la actividad de la AAG en muestras sanguíneas (pruebas de inhibición) y un consenso de un grupo de trabajo, con el objetivo de establecer un diagnóstico de laboratorio de la enfermedad de Pompe?

La prevalencia exacta de la enfermedad de Pompe en la población general es desconocida, y la frecuencia estimada varía entre las diferentes formas clínicas y grupos étnicos ${ }^{2,10}$. La prevalencia de la forma infantil tiene un rango que va de 1 en 33.333 a 1 en 138.000 , entre poblaciones taiwanesas y holandesas, respectivamente ${ }^{11,12}$. La prevalencia actual de la forma de comienzo tardío en Estados Unidos es desconocida. Un estudio en Nueva York sugiere que para todas las formas de presentación podría llegar a ser de 1 en $40.000^{13}$.

Existe un gran número de pacientes asintomáticos o con síntomas musculares leves, quienes presentan enzimas musculares elevadas, sin un diagnóstico definitivo. En un estudio reciente, las muestras de biopsia de tejido muscular, en pacientes con CK $>500 \mathrm{UI} / \mathrm{L}$ y sin signos de debilidad muscular, fueron revisadas y analizadas con tinción inmunohistoquímica. Ellos lograron un diagnóstico probable o definido en el $55 \%$ de los $\operatorname{casos}^{14}$. La alteración más frecuentemente identificada fue la enfermedad por depósito de glucógeno ${ }^{14}$. Se detectaron 4 casos de deficiencia de glucosidasa, arrojando una frecuencia de $3,8 \%{ }^{14}$. Este estudio mostró que, aún utilizando métodos sofisticados, un alto número de pacientes permaneció sin un diagnóstico definido.

Este estudio plantea el interrogante si la deficiencia de alfa-glucosidasa podría ser más frecuente de lo que se pensaba previamente, y sería la responsable de muchos casos de pacientes que se presentan con CK elevada de etiología no explicada.

No hay estudios que documenten la frecuencia de la enfermedad de Pompe en Argentina.

\section{Hipótesis de la investigación}

Se fundamenta en que la enfermedad de Pompe está subdiagnosticada en la población adulta. Existe un gran número de pacientes con elevación de enzimas musculares que no tienen un diagnóstico definitivo. Un importante porcentaje de esos pacientes podría tener una enfermedad de Pompe.

\section{Objetivos del estudio}

\section{Objetivo primario:}

Determinar la prevalencia de la enfermedad de Pompe entre pacientes mayores de un año con elevación persistente de CK de causa desconocida.

\section{Objetivo secundario:}

Describir las manifestaciones clínicas y demográficas de aquellos pacientes con CPK elevada.

\section{Materiales y métodos}

\section{Diseño del estudio}

Estudio de corte transversal en pacientes atendidos en un solo centro: Hospital Italiano de Buenos Aires.

\section{Criterios de inclusión}

Se incluyeron a todos los pacientes con aumento de CK en sangre, definida como la detección de al menos un valor $>500 \mathrm{UI} / \mathrm{L}$ y otro mayor a $200 \mathrm{UI} / \mathrm{L}$, durante un período mínimo de 1 año, entre los años 2010 y 2013, tomados de la base de datos del laboratorio del Hospital Italiano de Buenos Aires. 


\section{Criterios de exclusión}

Se excluyeron los pacientes bajo tratamiento actual con agentes hipolipemiantes; pacientes que fueron tratados con agentes hipolipemiantes, en quienes la CK se normalizó luego de suspender el tratamiento; pacientes que fueron tratados con agentes hipolipemiantes e interrumpieron el tratamiento durante un tiempo menor de 9 meses antes del período de inclusión y pacientes que reunieron criterios diagnósticos probables o definidos (criterios de Bohan \& Peter) para enfermedad muscular inflamatoria. Los pacientes con diagnóstico probable de miositis por cuerpo de inclusión (biopsia no típica) podrían ser incluidos.

A todos los pacientes incluidos se les realizó una evaluación clínica y estudios de laboratorio al momento de incluirlos en el estudio.

\section{Evaluación clínica}

La fuerza muscular se midió y graduó utilizando la escala de investigación médica (Medical Research Council Scale). Se evaluaron los músculos de extremidades superiores e inferiores y los músculos del cuello.

Se interrogó a los pacientes sobre los siguientes síntomas: dolores musculares, calambres, dolor lumbar bajo, cansancio, disnea, ortopnea, falta de descanso nocturno, despertares frecuentes, ronquidos, somnolencia diurna, cefaleas y letargo matutino.

\section{Pruebas de laboratorio}

Se midió la actividad enzimática AAG en muestras de sangre con el ensayo selectivo que utiliza el sustrato sintético 4-methylumbelliferyl- D-glucoside o glucógeno, en presencia de acarbosa y del ensayo de referencia enzimático para confirmar la reproducibilidad de la muestra 7. Todas estas mediciones fueron realizadas por Genzyme Argentina S.A.

\section{Consideraciones estadísticas}

\section{Análisis estadísticos:}

Se realizó un estudio descriptivo de las características demográficas y clínicas y de los resultados de las pruebas de laboratorio de todos los pacientes que presentaron elevación de CK, tomados de la base de datos del laboratorio y que cumplían con los criterios de inclusión.

Se determinó la prevalencia de la enfermedad de Pompe con su intervalo de confianza del $95 \%$.

\section{Resultados}

Se revisaron 849 historias clínicas y finalmente $199 \mathrm{pa}-$ cientes cumplieron con los criterios de inclusión.

En la actualidad pudimos estudiar a 110 pacientes. Se realizó el test enzimático en papel de filtro para enfermedad de Pompe en 93 pacientes.

La Tabla 1 describe las características demográficas y clínicas de los 93 pacientes.

\begin{tabular}{|l|c|}
\hline Características clínicas & \\
\hline Hombre / mujer & 69 / 24 \\
\hline Edad media & 54 años \\
\hline Antecedentes familiares de enf. muscular & $4(4,3 \%)$ \\
\hline Hipotiroidismo & $10(10,75 \%)$ \\
\hline Dislipemia & $44(47 \%)$ \\
\hline Enfermedad coronaria & $4(4,3 \%)$ \\
\hline Trasplante renal & $4(4,3 \%)$ \\
\hline Síntomas en la infancia & $9(9,6 \%)$ \\
\hline Dolor con el ejercicio & $47(50,53 \%)$ \\
\hline Dolor en reposo & $41(44 \%)$ \\
\hline Calambres & $72(77,4 \%)$ \\
\hline Disnea & $12(13 \%)$ \\
\hline Ortopnea & $6(6,5 \%)$ \\
\hline Ronquidos & $47(50,5 \%)$ \\
\hline
\end{tabular}

Tabla 1. Características demográficas y clínicas.

Un solo paciente presentó alteración del test enzimático en papel de filtro para enfermedad de Pompe, mostrando una prevalencia del 1,07\%. La alteración se confirmó con la determinación enzimática en leucocitos.

Al examen físico, 6 de 93 (5,58\%) pacientes presentaron debilidad leve en miembros superiores y 21 de 93 $(19,53 \%)$ pacientes presentaron debilidad en miembros inferiores de intensidad leve a moderada.

Se realizó electromiograma (EMG) en 21 pacientes: 10 EMG fueron normales, 5 EMG con patrón neuropático, 3 EMG con patrón miopático y 3 EMG con patrón mixto (Gráfico 1).

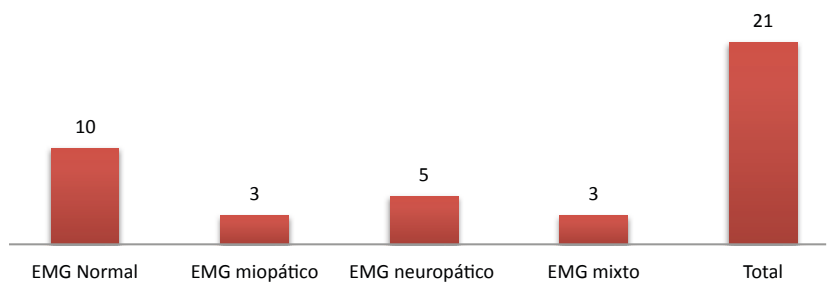

Gráfico 1. Patrones de EMG en los pacientes estudiados. 
Se realizó biopsia muscular en solo 10 pacientes: 5 biopsias normales, 3 biopsias con cambios inespecíficos, una biopsia con cambios miopáticos y otra con cambios neuropáticos (Gráfico 2).

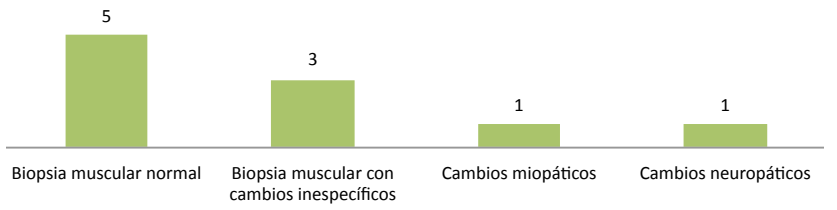

Gráfico 2. Hallazgos en las biopsias musculares.

Cuando se analizó la etiología del aumento de CK en los pacientes estudiados, sólo se pudo establecer un diagnóstico en el 34,4\% de los pacientes. Los mismos se encuentran desglosados en la Tabla 2.

\begin{tabular}{|l|c|}
\hline Diagnóstico $\mathbf{n}(\%)$ & $\mathbf{3 2 / 9 3}(\mathbf{3 4 , 4 \% )}$ \\
\hline Ejercicio & $4 / 32(12,5 \%)$ \\
\hline Enfermedad coronaria & $5 / 32(16 \%)$ \\
\hline Alcohol & $2 / 32(6 \%)$ \\
\hline Cirugía cardiovascular & $1 / 32(3 \%)$ \\
\hline Miositis infecciosa & $1 / 32(3 \%)$ \\
\hline Miocarditis & $1 / 32(3 \%)$ \\
\hline Hipokalemia & $1 / 32(3 \%)$ \\
\hline Trasplante renal & $4 / 32(12,5 \%)$ \\
\hline Polineuropatía & $1 / 32(3 \%)$ \\
\hline Poliradiculopatía desmielinizante crónica & $1 / 32(3 \%)$ \\
\hline Enfermedad motoneurona inferior & $1 / 32(3 \%)$ \\
\hline Distonía de torsión & $1 / 32(3 \%)$ \\
\hline Distrofia tipo 2 & $1 / 32(3 \%)$ \\
\hline Miopatía central core & $1 / 32(3 \%)$ \\
\hline Uso de corticoides & $7 / 32(22 \%)$ \\
\hline
\end{tabular}

Tabla 2. Etiología del aumento de CK en los pacientes estudiados.

\section{Discusión y conclusiones}

El objetivo de este estudio fue determinar la prevalencia de la enfermedad de Pompe y describir las características clínicas de los pacientes con CK elevada. Encontramos una baja prevalencia, similar a la publicada en la literatura. Se pudo establecer la etiología de los valores elevados de CK en un bajo porcentaje de los pacientes $(34,4 \%)$. Fue notable la poca utilidad que brindaron algunos métodos complementarios, como el EMG e incluso el número de biopsias musculares realizadas en el algoritmo de estudio de pacientes con CK elevados a lo largo de un año. Los informes de estos estudios tampoco fueron concluyentes y no aportaron demasiado para el diagnóstico etiológico de las alteraciones bioquímicas. Esto demuestra que, en general, esta patología se estudia poco y que el pronóstico de la misma es favorable ${ }^{15,16}$. Existe poca evidencia científica en relación al manejo de pacientes asintomáticos con CK elevada. Algunos estudios postulan que la CK elevada se define como un aumento $>1,5$ veces con respecto al valor de referencia ${ }^{17}$. Es importante descartar siempre las causas no neuromusculares para el diagnóstico diferencial ${ }^{18,19}$. Cuando sospechamos una etiología de índole muscular, debemos repetir la determinación de CK, previo a solicitar estudios invasivos. Una conducta apropiada en el estudio del paciente sería mantener al menos 7 días de reposo muscular y repetir 2 determinaciones de CK, separadas por el período de 1 mes. Si la CK persiste aún elevada, deberíamos realizar un EMG. La indicación de biopsia muscular se daría en los siguientes casos: EMG anormal, $\mathrm{CK}>3$ veces el valor normal, edad menor de 25 años o dolor provocado con el ejercicio ${ }^{17}$.

\section{Bibliografía}

1. Goldstein JL, Young SP, Changela M, et al. Screening for Pompe disease using a rapid dried blood spot method: experience of a clinical diagnostic laboratory. Muscle Nerve 2009; 40:32-6.

2. Diagnostic criteria for late-onset (childhood and adult) Pompe disease. Muscle Nerve 2009; 40:14960.

3. Kishnani PS, Steiner RD, Bali D, et al. Pompe disease diagnosis and management guideline. Genet Med 2006; 8:267-88.

4. Kishnani PS, Howell RR. Pompe disease in infants and children. J Pediatr 2004; 144:S35-43.

5. Winkel LP, Hagemans ML, van Doorn PA, et al. The natural course of non-classic Pompe's disease; a review of 225 published cases. J Neurol 2005; 252:875-84.

6. Koeberl DD, Kishnani PS, Chen YT. Glycogen storage disease types I and II: treatment updates. J Inherit Metab Dis 2007; 30:159-64.

7. Winchester B, Bali D, Bodamer OA, et al. Methods for a prompt and reliable laboratory diagnosis of 
Pompe disease: report from an international consensus meeting. Mol Genet Metab 2008; 93:275-81.

8. Van den Hout JM, Kamphoven JH, Winkel LP, et al. Long-term intravenous treatment of Pompe disease with recombinant human alpha-glucosidase from milk. Pediatrics 2004; 113:e448-57.

9. Kishnani PS, Corzo D, Nicolino M, et al. Recombinant human acid [alpha]-glucosidase: major clinical benefits in infantile-onset Pompe disease. Neurology 2007; 68:99-109.

10. Raben N, Plotz P, Byrne BJ. Acid alpha-glucosidase deficiency (glycogenosis type II, Pompe disease). CurrMol Med 2002; 2:145-66.

11. Chien YH, Chiang SC, Zhang XK, et al. Early detection of Pompe disease by newborn screening is feasible: results from the Taiwan screening program. Pediatrics 2008; 122:e39-45.

12. Ausems MG, Verbiest J, Hermans MP, et al. Frequency of glycogen storage disease type II in The Netherlands: implications for diagnosis and genetic counselling. Eur J Hum Genet 1999; 7:713-6.

13. Martiniuk F, Chen A, Mack A, et al. Carrier frequency for glycogen storage disease type II in New York and estimates of affected individuals born with the disease. Am J Med Genet 1998; 79:69-72.

14. Fernandez C, de Paula AM, Figarella-Branger D, et al. Diagnostic evaluation of clinically normal subjects with chronic hyperCKemia. Neurology 2006; 66:1585-7.

15. Jaap C. Reijneveld, Nicolette C. Notermans et al. Benign prognosis in idiopathic hyper-ck-emia Muscle Nerve 2000, 23:575-579.

16. Elisabetta D’Adda, Monica Sciacco, Maria Elisa Fruguglietti, et al. Follow-up of a large population of asymptomatic/oligosymptomatic hyperckemic subjects Journal of Neurology 2006, 253:1399-1403.

17. T. Kyriakides, C. Angelini, J. Schaefer, et al. EFNS guidelines on the diagnostic approach to pauci- or asymptomatic hyperCKemia European Journal of Neurology 2010, 17:767-773.
18. L. Morandi, C. Angelini, A. Prelle, et al. High plasma creatine kinase: review of the literature and proposal for a diagnostic algorithm Neurology Scince 2006, 27:303-311.

19. Bashar Katirji, FACP, and Mohamed M. Al-Jaberi, et al. Creatine Kinase Revisited Journal Clinic Neuromuscular Disease 2001; 2:158-163. 